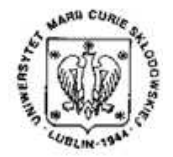

Annales UMCS Informatica AI XII, 1 (2012) 7-16 DOI: $10.2478 /$ v10065-012-0016-0

\section{Annales UMCS}

Informatica

Lublin-Polonia

Sectio AI

http://www.annales.umcs.lublin.pl/

\title{
Analysis of lung auscultatory phenomena using the Wigner-Ville Distribution
}

\author{
Mariusz Maciuk ${ }^{1,5 *}$, Wiesława Kuniszyk-Jóźkowiak ${ }^{1,2 \dagger}$, Anna Doboszyńska ${ }^{3 \ddagger}$, \\ Marta Maciuk ${ }^{4 \S}$ \\ ${ }^{1}$ Institute of Computer Science, Maria Curie-Skłodowska University \\ pl. M. Curie-Skłodowskiej 1, 20-036 Lublin, Poland \\ ${ }^{2}$ Department of Biomechanics and Computer Science, \\ External Faculty of Physical Education in Biała Podlaska \\ Akademicka 2, 21-500 Biała Podlaska, Poland \\ ${ }^{3}$ Clinical Nursing Department, Medical University of Warsaw \\ Ciotka 27, 01-445 Warsaw, Poland \\ ${ }^{4}$ Institute of Precision and Biomedical Engineering, \\ Warsaw University of Technology \\ Św. Andrzeja Boboli 8, 02-525 Warsaw, Poland \\ ${ }^{5}$ The State School of Higher Education in Chetm \\ Pocztowa 54, 22-100 Chetm, Poland
}

\begin{abstract}
In this paper the authors will try to discuss the applicability of Wigner-Ville Distribution for the digital analysis of auscultatory sounds. First of all, thte issues related to computer aided diagnosis are presented. Next, the methodology of research is shown and subsequently, the types of sounds are described. Another element of this work is the presentation of issues related to the digital signal processing including the Short-Time Fourier Transform(STFT), Wigner-Ville Distribution (WVD), and its variation- Smoothed Wigner-Ville Distribution (SWVD). This paper summarizes the results obtained using STFT and SWVD, showing SWVD more useful to detect the type of auscultatory sounds.
\end{abstract}

\footnotetext{
*mmaciuk@gmail.com

†jozkowiak@gmail.com

‡anna.doboszynska@wum.edu.pl

${ }^{\S}$ martamaciuk@gmail.com
} 


\section{Introduction}

The introduction of new technologies in medicine enabled fairly detailed examination of the human body. Owing to science we are able to describe the work of various organs and explain the processes taking place in them. A great number of tools have been created and used by doctors for diagnosis. With heart disease the diagnosis can be supported by resting or effort electrocardiogram tests, and even with a 24hour heart rate recording. It is here that expert systems have been developed which detect anomalies and present the most disturbing parts of the electrocardiogram for the doctor's final analysis. This development has significantly increased the percentage of detection of diseases at an early stage, and thus also increased the number of cured patients.

If we consider primary care physicians, it seems that the stethoscope is their primary tool. This examination involving listening to lung / heart sounds is the most frequently performed. On its basis a physician is able to determine the most common respiratory diseases. The amazing thing about it is that examination involves the tool, which has been present in medicine for over a century.

So far various efforts have been undertaken to create mechanisms that would facilitate doctors diagnose of lung pathology with the use of recorded sounds. In some studies neuron networks were used to recognize the characteristic patterns obtained from the Fourier Analysis [1] or Wavelet Analysis [2]. Other studies were carried out on the methods for detecting crackles on the basis of oscilloscope result shape analysis method.

The authors of this paper will attempt to discuss the use of the Wigner Ville Distribution in the analysis of auscultatory sounds and indicate its advantages with respect to the classical Fourier Analysis [3].

\section{The research methodology}

For the analysis of auscultatory sounds a test set was used, which allowed recording audio samples and their cataloging. With its help the data acquisition was done covering the sounds of healthy lungs and the most common illnesses in an outpatient settings. The test set consists of an electronic stethoscope 3M ${ }^{\mathrm{TM}}$ Littmann ${ }^{\circledR}$ Electronic Model 4100WS and PocketPC device (HP iPAQ HW 6915). Stethoscope can record 8-second track in one of three frequencies: enhanced mode $(20 \mathrm{~Hz}-2 \mathrm{kHz})$, funnel mode $(20 \mathrm{~Hz}-$ $200 \mathrm{~Hz})$ and diaphragm mode $(100 \mathrm{~Hz}-500 \mathrm{~Hz})$. The files saved by stethoscope have the original format e4k. Only with the application provided by the manufacturer of the stethoscope, the files are converted to the wave format with the following parameters: $128 \mathrm{~kb}$ / s, 16 bits per sample, mono, $8 \mathrm{kHz}$ sampling frequency. Such a file undergoes analysis by an application created in Matlab. 


\section{The classification of lung auscultatory sounds}

We distinguish two basic types of auscultatory sounds: physiological and additional. The first appear in normal healthy lungs and the other sounds are indication of pathologies.

Depending on the location of auscultation, physiological sounds are divided into normal vesicular sounds and bronchial breath sounds. The former occur as a result of air coming into the vesicle. Bronchial breath sounds are only heard over the trachea or large bronchi - a sound with a high frequency spectrum. It is created by the dynamic flow of air through a healthy bronchus We distinguish two basic types of auscultatory sounds: physiological and additional. The first appear in normal healthy lungs and the other sounds are indication of pathologies [4].

The group of additional auscultatory sounds includes: crackles, fine crackles and coarse crackles. Generally, crackles are short and voiceless sounds. They are formed by the sudden leveling of gas pressure caused by unblocking of small airway. Fine crackle sounds are commonly called crepitation, they are the sounds of higher frequency. Coarse crackles sounds are of lower frequency. Wheezes and rhonchi sounds are voiced. Wheezes are produced by the turbulent flow of air through the narrowed airways. These are the sounds of higher frequencies. Rhonchi are the sounds of lower frequencies caused by the presence of discharge in the airways. The last group according to the new classification is pleural rub. This is the effect of rubbing of parietal and lung pleural plaques $[5,6]$.

The next section will present the most popular tools for signal analysis, Fourier Transform and Wigner-Ville Distribution.

\section{Methods of Frequency Analysis of Signals}

\subsection{Fourier Transform}

Fourier transformation is the transformation of the signal $x(t)$ from the domain of time to the domain of frequency, described by the formula:

$$
X(\omega)=\int_{-\infty}^{\infty} x(t) e^{-j \omega t} d t .
$$

The result transform $X(\omega)$, called the spectrum contains information about the number of complex harmonic $e^{j 2 \pi f t}=\cos (2 \pi f t)+j \sin (2 \pi f t)$ of specific frequency in a tested signal. It is used as the primary tool for frequency analysis of signals.

This operation has its counterpart in the time domain, we can losslessly reconstruct the original signal by using the so-called Reverse Fourier Transform defined by the formula:

$$
x(t)=\frac{1}{2 \pi} \int_{-\infty}^{\infty} X(\omega) e^{j \omega t} d \omega .
$$


From a theoretical point of view in order to obtain the Fourier transform signal $x(t)$, it has to meet the so-called Dirichlet conditions on any boundary interval:

- the function is absolute and integral $\int_{-\infty}^{\infty}|x(t)| d t<\infty$

- has a finite value of maxima and minima in any finite interval,

- has a finite number of discontinuity points in any finite interval.

When analyzing real signals, it is assumed that the above conditions are met.

When analyzing signals in practice, we deal with discrete not continuous signals. A tool for working with the sampled signal $x(n)$ is called Discrete Fourier Transform $X(k)$ described by the formula:

$$
X(k)=\frac{1}{N} \sum_{n=0}^{N-1} x(n) e^{-j \frac{2 \pi}{N} k n}
$$

and inverse of this transform:

$$
x(n)=\sum_{k=0}^{N-1} X(k) e^{j \frac{2 \pi}{N} k n}
$$

where: $k$ - the harmonic number, $n$ - the number of signal samples, $x(n)$ - the value of signal samples, $N$ - the number of samples.

The above definitions present that the signal $x(n)$ and its spectrum $X(k)$ are the periodic functions of the base period $N$.

\section{The Time-Frequency Analysis of Signals}

When examining the real signals an important thing to remember is to give them the best character in the frequency and time domain. We need a way to determine the location of frequency components in time, in other words, the observation of signal spectrum variation in time. The signals used are non-stationary (variable spectrum). Therefore, the time-frequency methods were stressed when analyzing them.

\subsection{Spectrogram}

Spectrogram is one of the most common methods of signal analysis in the domain of time-frequency. This is the amplitude spectrum chart of signal for any time $t$, for which the signal is determined. The diagram of spectrogram is usually presented as a colourful map of the amplitudes, stretched in time and frequency domain.

Theoretical basis of spectrogram is Short-Time Fourier Transform, described by the formula:

where:

$$
S^{(h)}(t, f)=\int x(\tau) h^{*}(\tau-t) e^{-j 2 \pi \tau} d \tau
$$

$x$ - the analyzed signal

$h$ - the time window function. 
Spectrogram itself is expressed as a square module of STFT Short-Time Fourier Transform, that is:

$$
S P(t, f)=|\operatorname{STFT}(t, f)|^{2}=\left|\int x(\tau) h^{*}(\tau-t) e^{-j 2 \pi \tau} d \tau\right|^{2} .
$$

Since the time window depends only on one parameter (time), then it is difficult to choose exactly its size. To increase the resolution in the time domain (by reducing the window size) the frequency domain information is lost, and in order to obtain good resolution in the frequency domain (large time window) the resolution in the time domain deteriorates.

\subsection{Wigner-Ville Distribution}

Wigner-Ville Distribution (WV) is a tool for analyzing non-stationary signals, defined by Edgue Wigner in 1932 and developed by Jean-Andre Ville'aw in 1948 [7]. In fact, it shows the distribution of energy density and in terms of information contained it can be compared to the spectrogram. Similarly to the signal of amplitude spectrum chart, the graph WV is usually presented as a colourful map of spectra produced during the transformation. The WV distribution perfectly imitates frequency (TF) in the spacetime, a linear change in frequency [8]. It belongs to a broad class of distributions, called the Cohen's class.

The main idea of this transformation is an expression of measure of local symmetry of the signal at any point in the time-frequency plane. It is defined as follows:

$$
\begin{gathered}
S_{x}^{W V}(t, f)=\int_{-\infty}^{\infty} x\left(t+\frac{\tau}{2}\right) x^{*}\left(t-\frac{\tau}{2}\right) e^{-j 2 \pi f \tau} d \tau \\
S_{X}^{W V}(t, f)=\int_{-\infty}^{\infty} X\left(f+\frac{\nu}{2}\right) X^{*}\left(f-\frac{\nu}{2}\right) e^{-j 2 \pi f \nu} d \nu
\end{gathered}
$$

where $x(t)$ is the real signal (in the definition of the Wigner) or analytic (in the definition Ville). The representation of the Wigner-Ville Distribution is the Fourier Transform containing the so-called WV kernel with respect to the variable $\tau$ or $\nu$ :

$$
\begin{gathered}
x x(t, \tau)=x\left(t+\frac{\tau}{2}\right) x *\left(t-\frac{\tau}{2}\right) \\
X X(f, \nu)=X\left(f+\frac{\nu}{2}\right) X *\left(f-\frac{\nu}{2}\right) .
\end{gathered}
$$

The representation of the Wigner-Vile'a has the highest concentration of energy in the time-frequency space, which means that this method gives the best overall resolution. Additionally, it transfers the information in the signal carrier wave very well. But when analyzing certain signals, there may be some components that effectively make it difficult to interpret the result. They are caused by mutual overlapping of spectral components. This is an oscillatory phenomenon. Therefore, it is easier to use an analytical representation of the signal. 
Bythe analytic signal $s_{a}(t)$ we mean a representation of the real signal $s(t)$, in which the real part is the signal $s(t)$, while the imaginary part is presented as the Hilbert Transform of this signal.

$$
s_{a}(t)=s(t)+j \hat{s}(t)
$$

where $\hat{s}(t)$ is convolution of the functions $s(t)$ with the function $h(t)=\frac{1}{\pi t}$

$$
\hat{s}(t)=h(t) * s(t) .
$$

The use of such a solution enables to reset the TF spectrum for negative frequencies and complete removal of parasitic interference between the components of negative and positive frequencies.

The digitized form of the Wigner-Ville Distribution is defined as follows:

$$
S_{x}^{W V}(n, k)=2 \sum_{m=-\infty}^{+\infty} x(n+m) x^{*}(n-m) e^{-j\left(\frac{4 \pi}{N} k\right) m}
$$

for $k=0,1,2, \ldots, N / 2-1$.

Because of sampling of the signal with an inappropriate frequency, the phenomena of aliasing may appear. Aliasing is the irreversible distortion of signal in the process of sampling resulting from the failure to meet the assumptions of Kotielnikowa-Shannon Theorem (Nyquist-Shannon Sampling Theorem) Distortion is manifested by the presence of false frequencies in the signal components(aliases). Sampling should be done by choosing the signal sampling frequency fs meeting the Nyquist condition. This condition states that the fs of a given signal should be at least twice the size of maximum frequency present in the signal [9].

The so-called cross-spectra are formed as a consequence of the fact that the Wigner representation is nonlinear (bilinear), which means that the sum of the Fourier spectra is not equal to the sum of the Fourier Transform of two signals.

$$
S_{x_{1}, x_{2}}^{W V}(t, f)=S_{x_{1}}^{W V}(t, f)+S_{x_{2}}^{W V}(t, f) .
$$

The cross interferences are oscillatory and are present between the spectra of individual components of their own signals. Their period is equal to the inverse of the distance between these components in the space TF [10].

This is the basic drawback of this method, which can be reduced through the additional smoothing of the signal.

\subsection{Smoothed Wigner-Ville Distribution (SWVD)}

The two basic and major issues related to the representation of the Wigner-Ville are negative values in the spectrum and the emergence of cross-interference. To get rid of harmful, parasitic interference in the received time-frequency spectral matrix, interference blanking is used with smoothing windows. In fact, this operation has little effect on the signal itself, but weakens occurring interferences significantly due to 
their oscillatory nature. This effect may lead to slight blur (widening) of the spectrum. Time-frequency signal smoothing makes the transforms result more readable and easier to interpret [11]

In practice, it is advisable to use separate window smoothing in the time and frequency domain. In the case of the transform of the continuous time, the interpretation of the windows is as follows:

$$
F(t, \nu)=g(t) H(\nu)
$$

where

$$
\iint F(t, \nu) d t d \nu=1
$$

SWVD is defined as follows

$$
S W V D_{x}(t, \nu)=\iiint_{-\infty}^{\infty} x\left(\theta+\frac{\tau}{2}\right) x^{*}\left(\theta-\frac{\tau}{2}\right) g(t-\theta) H(\nu-w) e^{-j 2 \pi w \tau} d w d \theta d \tau
$$

Finally, we get:

$$
S W V D_{x}(t, \nu)=\iint_{-\infty}^{\infty} x\left(\theta+\frac{\tau}{2}\right) x^{*}\left(\theta-\frac{\tau}{2}\right) g(t-\theta) h(\tau) e^{-j 2 \pi \nu \tau} d \theta d \tau .
$$

From the above formula it can be easily seen that the signal $x(t)$ is separately smoothed in the time and frequency domain. Characteristics of smoothing can therefore be controlled independently along two axes. Smoothing in the frequency domain controlled by means of the window h allows the removal of interference that occur along the time axis. However, the interference on the frequency axis can be smoothed by manipulating the window g, modified in the time domain.

The smoothed time-frequency representation of Wigner-Ville has a unique role in the analysis of frequency of non-stationary signals, especially biomedical signals, where it is important to reproduce any information which can be collected.

\section{Implementation}

Application of time-frequency analysis of lung sounds has been developed in the Matlab environment. The script allows to select the file for analysis and the setting of width as well as a time window. In order of standardization the article set the window width to 2048 samples, and as a window there was adopted the Hamming time window, defined as follows:

$$
w(n)=0.53836-0.46164 \cos \left(\frac{2 \pi n}{N-1}\right) .
$$

The window was shifted by half its width. The graphs obtained in the z-axis are shown in a logarithmic scale. 
Pobrane z czasopisma Annales AI- Informatica http://ai.annales.umcs.pl

Data: 26/04/2023 12:40:43

\section{$7 \quad$ Results}

The model files were subjected to the Fourier Analysis (left column) and presented in the spectrograms. Also, the same recordings were presented as the result of Smoothed Wigner-Ville Distribution (right column). Gray level represents the value of distribution in $\mathrm{dB}$.
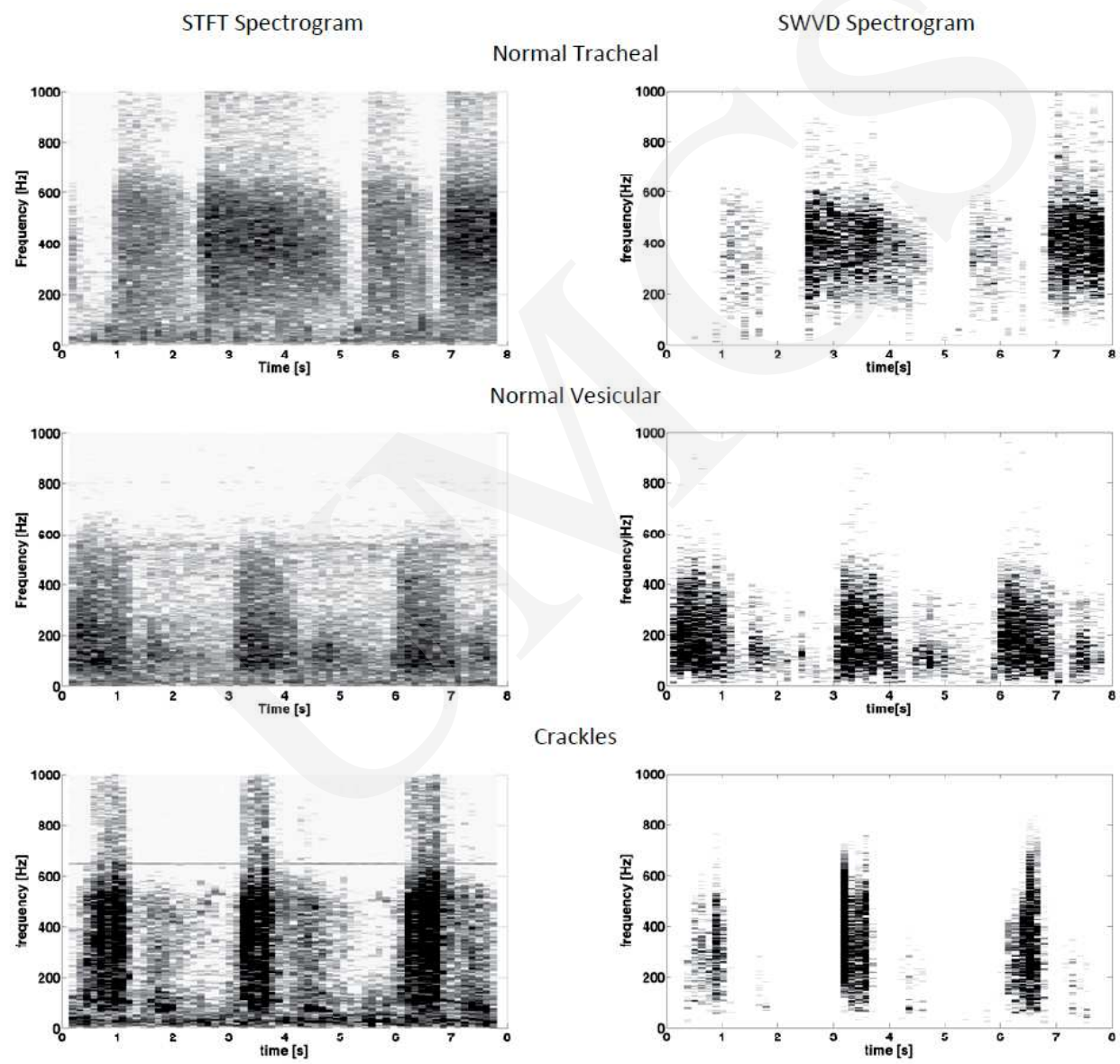

Crackles

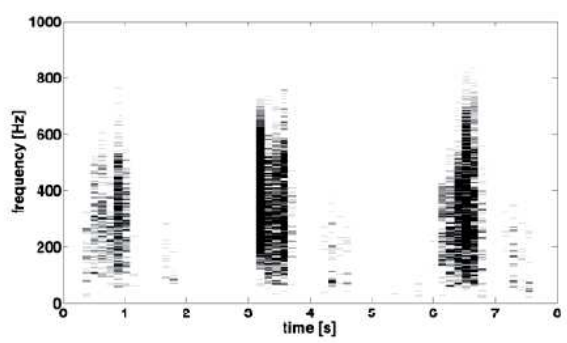

The above statement allows us to compare the obtained results. At the first glance we can tell the difference - the data obtained by applying SWVD is more readable and thus easier to interpret.

By making a simple characterization, we can distinguish the correct sounds - they are characterized by a marked breath phase. On the diagrams these are the areas recorded over a longer time space and over a higher frequency range. The sounds recorded from the area of the trachea / bronchus are noticeable at higher frequencies than the sounds recorded from the area of the base of the lungs. 
Pobrane z czasopisma Annales AI- Informatica http://ai.annales.umcs.pl

Data: 26/04/2023 12:40:43
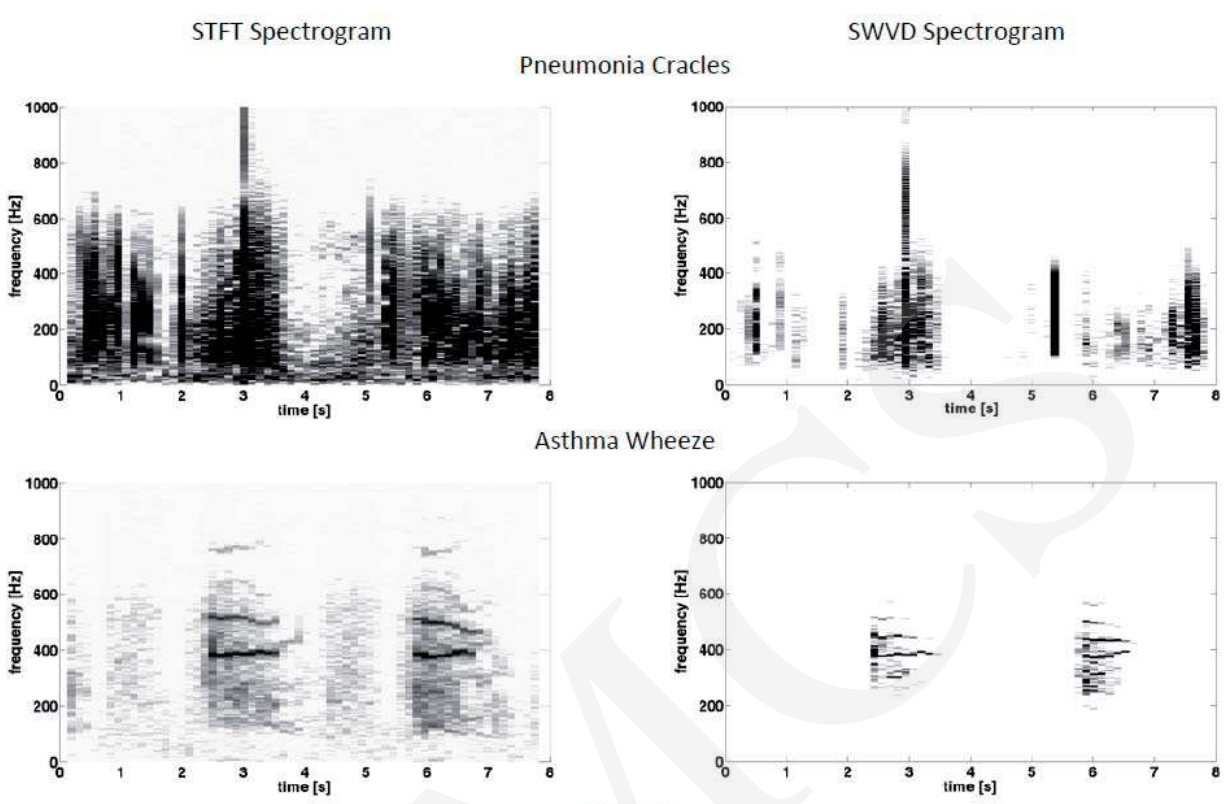

Rhonchi
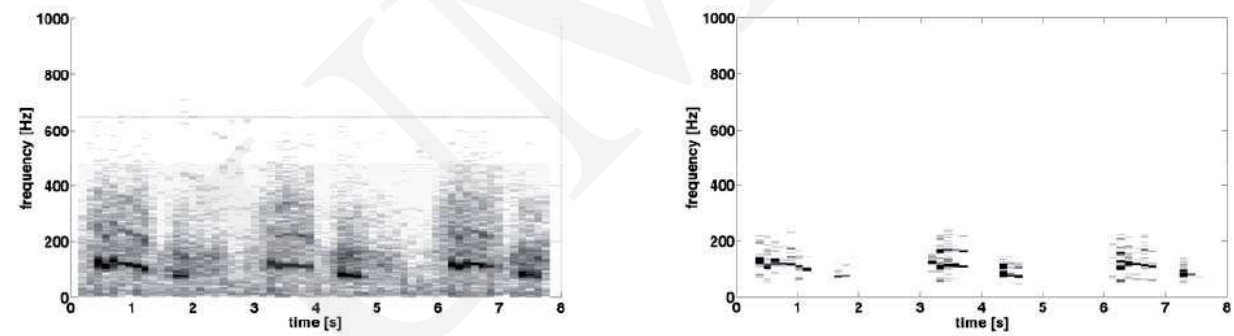

Auscultatory sounds containing crackles can be identified by short-term but dynamic, recorded in a very wide frequency range of crackle sounds. On the diagram they are represented by vertical peaks.

Wheezes shown in the graphs are derived from a patient with asthma. They and the rhonchi sounds can be recognized by the horizontal "waves" on the spectrogram. Wheezes differ from the rhonchi sounds in the range of frequencies they are recorded. Wheezes are of a high frequency about $400-600 \mathrm{~Hz}$, rhonchi of a small one about 100$200 \mathrm{~Hz}$.

\section{Conclusions}

The use of Wigner Ville Distribution smoothed by the Hamming window in the analysis of auscultatory sounds brings us closer to create a system that can automatically detect the type of recorded sound. This application would allow patients to perform 
Pobrane z czasopisma Annales AI- Informatica http://ai.annales.umcs.pl

Data: 26/04/2023 12:40:43

self-monitoring of their lungs at home without help of a doctor - and with detection of pathological signals it would recommend consultation with a specialist.

\section{References}

[1] Goswami J. C., Chan A. K., Fundamentals of Wavelets Theory, Algorithms and Application, Wiley (2011).

[2] Zieliński T. P., Digital signal processing : from theory to applications, in Polish Cyfrowe Przetwarzanie Sygnałów - Od teorii do zastosowań, Warszawa: WKŁ (2009).

[3] Claasen T. A. C. M., Mecklenbrauker W. F. G., The Wigner - Ville Distribution - A Tool for Time - Frequency Signal Analysis. Part I: Continuous Time Signals, Philips J. Res. 35 (1980): 217.

[4] Szczeklik A., Choroby wewnętrzne - Stan wiedzy na rok 2010, Kraków: Medycyna Praktyczna (2010).

[5] Andre Q., Time-Frequency Analysis, Digital Signal Processing Using Matlab, Wiley (2008): 279.

[6] Maciuk M., Kuniszyk-Jóźkowiak W., Kuć K., Analysis of respiratory sounds, in Polish: Analiza Fenomenów Osłuchowych, Scientific Bulletin of Chełm, Section of Techical Sciences, PWSZ Chełm 1 (2008).

[7] Pasterkamp H., Kraman S. S., Wodicka G. R., Respiratory Sounds - Advanced Beyond Stethoscope, American Journal of Respiratory and Critical Care Medicine 156 (1997): 974.

[8] Dokur Z., Olmez T., Clasification of Respiratory Sounds by Using an Artificial Neural Network, International Journal of Pattern Recognition (2003): 567.

[9] Vanuccini L., Rossi M., Pasquali G., A new method to detect cracles in respiratory sounds, Technology and Health Care 6 (1998): 75.

[10] Kandaswamy A., Kumar C. S., Ramanathan R. P., Jayaraman S., Malmurugan N., Neural classification of lung sounds using wavelet coefficients, Computers in Biology and Medicine 34 (2004): 523.

[11] Ville J., Theorie et applications de la notion de signal analitique, Cables et Transmissions 2A (1) (1948): 61. 